\title{
Study of the one-way speed of light anisotropy with particle beams
}

\author{
Bogdan Wojtsekhowski ${ }^{1, \star}$ \\ ${ }^{1}$ Thomas Jefferson National Accelerator Facility, Newport News, Virginia 23606 USA
}

\begin{abstract}
Concepts of high precision studies of the one-way speed of light anisotropy are discussed. The high energy particle beam allows measurement of a one-way speed of light anisotropy (SOLA) via analysis of the beam momentum variation with sidereal phase without the use of synchronized clocks. High precision beam position monitors could provide accurate monitoring of the beam orbit and determination of the particle beam momentum with relative accuracy on the level of $10^{-10}$, which corresponds to a limit on SOLA of $10^{-18}$ with existing storage rings. A few additional versions of the experiment are also presented.
\end{abstract}

\section{Introduction}

A constant and isotropic speed of light is a key postulate of the special relativity theory, SR, as formulated by A. Einstein in 1905 [1]. In a search for a light related medium, ether, A. Michelson [2] developed an extremely sensitive method of measurement of the anisotropy of the two-way speed of light, $c_{2}$, which is an average of the speeds in two opposite directions. A modern experiment of this type [3] reached an extremely high precision, $10^{13}$ times higher than was obtained in the Michelson-Morley experiment [4], and found that $c_{2}$ is isotropic at least to the level of $10^{-18}$. The one-way speed of light anisotropy tells us about the directional variation of a difference in the speed of light in two opposite directions. Current studies of SOLA are motivated by predictions in string and quantum gravity theories [5], as well as by general interest in physics beyond the Standard Model. The one-way speed of light, $c_{1}$, could be measured by using two synchronized clocks. However, there is a very significant difficulty in realizing clock synchronization at the required precision. If observed, variation of the speed of light could be an indication of a quantum gravity effect in the laboratory.

Several tests have been proposed for the study of $c_{1}$ which do not require clocks because they address the difference between the speed of light at different polarizations or the difference between the speed of light and the speed of electrons in the same direction. The latter could be explored using threshold reactions such as vacuum Cherenkov radiation and photon decay to an electronpositron pair [6]. The maximum attainable speed of electrons could also have directional anisotropy. A Compton process was used recently in an experiment [7], which provided a constraint on a sidereal time variation of a combination of the maximum attainable speed of electrons and the one-way speed of light. Many (but not all) the SOLA

\footnotetext{
^e-mail: bogdanw@jlab.org
}

experiments were analyzed in terms of mSME developed by A. Kostelecky and collaborators [8].

\section{Concept of the method}

The trajectory of the high energy beam in a transverse magnetic field provides an exciting opportunity to investigate SOLA [9]. Indeed, according to SR, the momentum of the particle is defined by the difference between its speed and a maximum attainable speed for the particle (assumed from now on to be equal to the speed of light). The relativistic expression for the particle momentum, $p=m v / \sqrt{(c-v) \cdot(c+v)}$, shows that when $v$ approaches $c$, the $p$ value is very sensitive to the variation of $(c-v)$. For a charged particle moving in a transverse magnetic field, according to the conventional Lorentz force, we should have a constant absolute value of the velocity vector $\vec{v}$. If the speed of light, $c_{1}$, varies, the experimental observable, a particle momentum, will also change with sidereal time and its relative variation would be enhanced by a factor of $\gamma^{2}$.

Many modern accelerators are equipped with beam position monitors which have an accuracy of a few microns or better in a single measurement. Using a typical magnetic system with the dispersion function of a few meters, it should be possible to measure beam momentum to a few $10^{-6}$ precision many times per second. There are several accelerators with a beam gamma factor of $10^{4}$, which provides a boost factor of $10^{8}$ in sensitivity for the speed of light variation, which could as a result reach the quantum gravity domain whose possible onset is $10^{-17}$ [5].

In addition to a sidereal time variation, very short-term changes are also well motivated physics, e.g. transient-intime effects [10], and they could be detected via particle momentum changes in a high energy accelerator. Correlated measurements at several facilities could provide a realistic way to search for such a phenomenon [11]. 


\section{Beam experiment at CEBAF}

The CEBAF accelerator has ten $180^{\circ}$ magnetic arcs with beam energies varying from one to eleven $\mathrm{GeV}$. There are no accelerating cavities in the arcs, and in each arc the beam energy is constant (after correction for a small and stable synchrotron radiation effect). Formulated in the Ref. [9] plan is a measurement of the momentum of the beam at two points at the beginning and the end of each arc by using beam deflection in arc magnets and beam position monitors. The analysis of the beam momenta ratio at these two points as a function of time would be independent of beam energy variation, which is typically of $10^{-4}$.

The data for such an experiment would be collected during normal CEBAF operation for hadron physics experiments [12]. Similar measurements could be performed at the KEK high energy electron/positron rings and at LHC for protons, as well as at a number of $\mathrm{X}$-ray facilities.

The main limitation in such an experiment is due to instabilities in the magnetic system and beam line geometry due to a number of effects such as ground movement (a tidal effect), oscillation in power supplies for magnetic elements, daily variation of the tunnel temperature and many others. However, analysis of the data over many days should allow effective selection of the signal with sidereal periodicity.

\section{Beam experiments at positron/electron rings}

There are storage rings where one can take advantage of closely located orbits of counter-propagating beams and analyze the ratio of the beam momenta in which the instabilities of the magnetic system and geometry drifts are canceled out because they have identical effects on both beams [13].

Two electron/positron storage rings, CESR and VEPP4 , currently operate with a common magnetic system for both beams and have large beam gamma factors. In these storage rings, the electron-to-positron orbit differences, originating mainly from beam energy loss due to synchrotron radiation and coherent effects, are less than 0.5 $\mathrm{mm}$. Such a configuration of the experiment is able to probe the CPT-even terms of the Lorentz violation with precision limited only by the short term stability of the beam position monitors (assuming that the same BPMs and electronics are used from both beams).

Analysis of the first CESR data allows us to put a limit on $\delta c_{1} / c_{1} \leqslant 10^{-14}$. Currently, experiments are active in both accelerators $[14,15]$.

\section{Experiments at the X-ray synchrotron radiation storage rings}

There are several storage rings with multi GeV electron beams which are used as X-ray sources to do photon physics [16-18]. Each of them provides dozens of X-ray beam lines with very stable direction (on the order of 0.1 micro radian) and position (on the order of a micro meter) of photons at users' instruments.
The beam orbit in a typical X-ray storage ring is subject to regular corrections which allow the X-ray beam to be kept stable. These corrections complicate the use of the beam orbit data for the speed of light investigation. However, stabilization of the X-ray beam directions leads also to stabilization of the electron beam directions at the location of X-ray emission in the insertion devices.

As a result, the magnetic field integral on the beam orbit between two X-ray beam emission points would provide a perfect measure of the beam momentum and open the possibility of SOLA investigation at many synchrotron radiation facilities.

\section{Experiment with a muon storage ring}

A high precision measurement of the muon anomalous magnetic moment, the muon $(g-2)$ experiment, was performed at a specialized storage ring with decay in flight of $3.09 \mathrm{GeV} / \mathrm{c}$ muons [19]. It was done via analysis of the observed in the detectors muon decay rate with the 5-parameter fit: $N(t)=N_{0} e^{-(t / \gamma \tau)}\left[1+A \cos \left(\omega_{a} t+\phi\right)\right]$. The data were also used to evaluate possible Lorentz invariance violations in a muon sector of mSME [20]. In that analysis, the spin precession frequencies $\omega_{a}$ for positive and negative muons were analyzed for potential sidereal time variation.

The muon spin rotation frequency at a given magnetic field value is defined by the muon magnetic moment and its momentum. The relative angle between the momentum and spin directions is proportional to the anomaly magnetic moment and momentum rotation angle. The change in the observed muon decay rate between detectors could be used as a measure of the momentum rotation angle advance. As the speed of light variation changes the particle momentum, the local spin rotation frequency also varies. Assuming that the value of the muon momentum changes along the particle orbit, the effect of interest should be searched for as a function of the orbit phase in contrast to the performed analysis [20], where the frequency $\omega_{a}$ was averaged over all detectors along the orbit before a search for a realtime variation.

We propose that in the fit function above, the $\phi$ should be replaced by $\phi_{0}+B \times \cos (\Omega t+\psi)$, where $\Omega$ is the Earth rotation frequency and $\psi$ is a constant defined by the locations of the detector along the beam orbit and the laboratory. The proposed analysis will provide a limit on the parameter $B$ and, as a result, a first estimate for a sidereal time variation of the maximum attainable speed of a muon.

\section{Analysis of a resonance production rate}

The asymmetric electron-positron collider allows an interesting opportunity to monitor the speed of light by observing the production rate variation when the combined beam energy is set slightly off resonance [9]. In the case of $\Upsilon$ resonance the observed relative width is $\Gamma / \mathrm{M} \sim 10^{-3}$. Indeed, due to a large difference in gamma factors, the speed of light variation induces changes in the momentum for a high energy beam (h.e.b.) much larger than for a lower energy beam. 
As a result, the total energy in the lab system varies with sidereal time and the resonance production rate also varies. The size of the rate variation could be estimated as $\frac{\delta c_{1}}{c_{1}} \times \gamma_{\text {h.e.b. }}^{2} \times \mathrm{M} / \Gamma$. At the projected luminosity of Belle-2 experiment [21], statistics collected every day would be of $10^{8} \Upsilon$ events, which would correspond to a sensitivity for $\delta c_{1} / c_{1}$ on the level of $10^{-15}$.

\section{Polarized electron-positron beams at a storage ring}

The measurement of the electron and positron anomalous magnetic moments ratio, $\mu_{+}^{\prime} / \mu_{-}^{\prime}$, at VEPP-2m [22] found that precession frequencies are equal to each other at the relative level $1.2 \cdot 10^{-7}$ (a record precision for the time of that experiment). The current best limit for $\left(g_{+}-/ g_{-}\right) / g_{\text {aver }}$ of $2 \cdot 10^{-12}$ was obtained using the particle trap method [23].

As a result, we can consider [9] the data from the storage ring experiment as a comparison of the electron and positron beam momenta when the beams are moving in opposite directions in a transverse magnetic field and make an estimate of the achievable sensitivity for the $c_{1}$ variation on the level of $1 \cdot 10^{-13}$ from those 1987 data. Obviously, this could be improved by two to three orders of magnitude using a higher beam energy, e.g. at VEPP-4, and advanced spin resonance detection methods [24].

\section{Acknowledgments}

The author would like to thank the organizing committee of the "Advances in Dark Matter and Particle Physics" workshop for support and the invitation to give a talk. I greatly appreciate comments from D. Budker, Yu. Shatunov and V. Zelevinsky, and collaborations with W. Bergan, G. Franklin, V. Lebedev, S. Nikitin, G. Nowak, Y. Roblin, D. Rubin, B. Schmookler, and B. Vlahovic. This work was supported in part by the NCCU NSF CREST Center, and by Department of Energy (DOE) contract number DE-AC05-06OR23177, under which the Jefferson Science Associates operates the Thomas Jefferson National Accelerator Facility.

\section{References}

[1] A. Einstein, Annalen der Physik, 17, 891 (1905).

[2] A.A. Michelson, American Journal of Science, 22, 120 (1881).

[3] M. Nagel et al., Nature Communications, 6, 8174 (2015).

[4] A.A. Michelson and E.W. Morley, Am. J. Sci., 34, 333 (1887).

[5] D. Mattingly, Living Rev. Rel. 8, 5 (2005).

[6] M.A. Hohensee et al., Phys. Rev. Lett. 102, 170402 (2009).

[7] J.-P. Bocquet et al., Phys. Rev. Lett. 104, 241601 (2010).

[8] V.A. Kostelecky and N. Russell, Rev. Mod. Phys. 83, 11 (2011).

[9] B. Wojtsekhowski, Euro Physics Letters, 108, 31001 (2014); arXiv:1409.6373.

[10] M. Pospelov et al., Phys. Rev. Lett. 110, 021803 (2013).

[11] D. Budker and A. Derevianko, Physics Today 68(9), 10 (2015).

[12] B. Wojtsekhowski and Y. Roblin, proposal PR12-15002, JLab PAC43, 2015.

[13] B. Wojtsekhowski, arXiv:1509.02754.

[14] D. Rubin et al., private communication, Aug. 2016.

[15] S. Nikitin et al., private communication, Oct. 2016.

[16] http://www.spring8.or.jp/en/

[17] https://www1.aps.anl.gov

[18] http://www.lightsources.org/facility/esrf

[19] G.W. Bennett et al., Phys. Rev. D 73, 072003 (2006).

[20] G.W. Bennett et al., Phys. Rev. Lett. 100, 091602 (2008).

[21] T.E. Browder et al., arXiv:0802.3201.

[22] I.B. Vasserman et al., Phys. Lett. B, 198302 (1987).

[23] C. Patrignani et al.. (Particle Data Group), Chin. Phys. C, 40, 100001 (2016).

[24] Yu.M. Shatunov, private communication, Sept. 2016. 